\title{
A Convergence Result for the Kuramoto Model with All-to-All Coupling*
}

\author{
Mark Verwoerd ${ }^{\dagger}$ and Oliver Mason ${ }^{\dagger}$
}

\begin{abstract}
We prove a convergence result for the standard Kuramoto model with all-to-all coupling. Specifically, we show that the critical coupling strength associated with the onset of completely phase-locked behavior converges in probability as the number of oscillators tends to infinity.
\end{abstract}

Key words. coupled oscillators, synchronization, phase-locking, Kuramoto model, convergence

AMS subject classifications. 34C15, 35B $32,37 \mathrm{C} 25$

DOI. $10.1137 / 090771946$

1. Introduction. The Kuramoto model of a system of weakly coupled nonlinear oscillators on a complete graph $K_{n}, n \geq 2$, is given by $[1,2,3,4]$

$$
\dot{\theta}_{i}=x_{i}+\frac{k}{n} \sum_{j=1}^{n} \sin \left(\theta_{j}-\theta_{i}\right), \quad i=1, \ldots, n,
$$

where $\theta_{i}(\cdot) \in \mathbb{R}$ and $x_{i} \in \mathbb{R}$ denote the phase and intrinsic (or natural) frequency, ${ }^{1}$ respectively, of oscillator $i$, and $k \in \mathbb{R}_{\geq 0}$ denotes the coupling coefficient or coupling strength. The behavior of system (1) depends strongly upon the value of the coupling strength, as described in [3] and elsewhere. At low values of $k$, the oscillators tend to oscillate more or less independently, and little or no coherent behavior is observed. As $k$ is gradually increased, the oscillators continue to oscillate incoherently for a while but then slowly lock into step as $k$ exceeds the threshold value defined by the "Kuramoto coupling" $[2,5,6,7]$. (For a symmetric unimodal distribution of intrinsic frequencies, the value of the Kuramoto coupling (in the limit as $n \rightarrow \infty$ ) is given by $k=\frac{2}{\pi g(\mu)}$, where $g$ is the underlying density function and $\mu$ is the first moment of $g$ [3].) From this point on, more and more oscillators join the cluster of locked oscillators, until eventually a state is reached in which all the oscillators are (pairwise) phase-locked. The value of the coupling that defines this final transition to a global (or completely) phase-locked state is referred to here as the critical coupling and is denoted by $k_{\mathrm{c}}$. The present paper is concerned with the behavior of $k_{\mathrm{c}}$ in the limit as the number of oscillators $(n)$ tends to infinity.

Of the two transitions mentioned above, the first transition from incoherent to partially coherent behavior, has generally received most attention, owing perhaps to its more immediate physical significance. One drawback that presents itself when studying the first transition

\footnotetext{
${ }^{*}$ Received by the editors September 23, 2009; accepted for publication (in revised form) by A. Stuart June 6 , 2011; published electronically September 13, 2011.

http://www.siam.org/journals/siads/10-3/77194.html

${ }^{\dagger}$ The Hamilton Institute, National University of Ireland, Maynooth, County Kildare, Ireland (mark.verwoerd@ nuim.ie, oliver.mason@nuim.ie).

${ }^{1}$ The reason we use $x_{i}$ rather than the more common notation $\omega_{i}$ to denote the intrinsic frequency is that, in keeping with standard probabilistic notation, the latter symbol is used here to define a probabilistic event.
} 
is that it is hard to characterize mathematically. For this reason, an approach based on limits such as developed here for the second transition would fail when applied to the first transition. The difficulty with the first transition is that there is no obvious critical phenomenon in the finite-dimensional Kuramoto model that one can associate with the transition from incoherent to partially coherent behavior. For the second transition, such a critical phenomenon does exist and is easily defined. Indeed, for this case the critical phenomenon is precisely the emergence of a (completely) phase-locked solution which defines the critical value of the coupling coefficient, $k_{\mathrm{c}}$. In the present paper we show that the random variable $k_{\mathrm{c}}$, which generally depends upon the particular realization of intrinsic frequencies, converges in probability as the number of oscillators tends to infinity. To the best of our knowledge, this is the first convergence result for the classical Kuramoto model. A related result for the Winfree model was obtained in $[7]$.

There are many papers that deal directly or indirectly with the second transition; we mention some recent contributions most relevant to the present work. References [8] and [9] provide a detailed account of the spectral properties of the phase-locked state $\left(k \geq k_{\mathrm{c}}\right)$ and the partially phase-locked state $\left(k<k_{\mathrm{c}}\right)$, respectively. In reference [10], the authors of the present paper describe a numerical algorithm for computing $k_{\mathrm{c}}$ given any finite realization of intrinsic frequencies [10] (see also Appendix A). An extension of this result to the case of a complete bipartite graph is described in the follow-up paper [11].

The structure of this paper is as follows. In sections 2 and 3 we fix our notation and review relevant background material. The main result is presented in section 4 , and this is followed by a discussion of its applications in section 5. Section 6 closes with conclusions.

\section{Mathematical preliminaries.}

2.1. Notation. Throughout the paper, $\mathbb{R}(\mathbb{C})$ denotes the field of real (complex) numbers, $\mathbb{R}_{\geq 0}$ denotes the set of all nonnegative real numbers, and $\mathbb{N}$ denotes the set of positive integers. For $v \in \mathbb{R}^{n}$ and $1 \leq i \leq n, v_{i}$ denotes the $i$ th component of $v$. For any real number $x,|x|$ denotes the absolute value of $x$. For any $n$-tuple $x=\left(x_{1}, x_{2}, \ldots, x_{n}\right)$, the notation $\langle x\rangle_{n}$ denotes the arithmetic mean of $x$; that is, $\langle x\rangle_{n}:=\frac{1}{n} \sum_{j=1}^{n} x_{n}$.

2.2. Probability. The terminology and notation adopted here is standard. For background on basic probability theory, the interested reader should consult [12]. Let $(\Omega, \mathcal{F}, \mathbb{P})$ be a probability space. A random variable is a function $X: \Omega \mapsto \mathbb{R}$ with the property that $\{\omega \in \Omega: X(\omega) \leq x\} \in \mathcal{F}$ for each $x \in \mathbb{R}$. We say that $X$ is continuous if its distribution $F(x)=\mathbb{P}(X \leq x)$ can be written as

$$
F(x)=\int_{-\infty}^{x} f(u) \mathrm{d} u
$$

for some integrable $f: \mathbb{R} \mapsto[0, \infty)$. The function $f$ is called the (probability) density function of $X$. The expectation of a continuous random variable $X$ with density function $f$ is given by

$$
\mathbb{E} X=\int_{-\infty}^{\infty} u f(u) \mathrm{d} u
$$

whenever this integral exists. Two random variables $X$ and $Y$ are independent if $\{X \leq x\}$ and $\{Y \leq y\}$ are independent events for all $x, y \in \mathbb{R}$. If $X$ and $Y$ are independent, then for 
all $a, b \in \mathbb{R}$ we have that $\mathbb{E}(a X+b Y)=a \mathbb{E} X+b \mathbb{E} Y$. Let $X, X_{1}, X_{2}, \ldots$ be random variables on some probability space $(\Omega, \mathcal{F}, \mathbb{P})$. We say that $X_{n} \rightarrow X$ almost surely, written $X_{n} \stackrel{\text { a.s. }}{\rightarrow} X$, if $\left\{\omega \in \Omega: X_{n}(\omega) \mapsto X(\omega)\right.$ as $\left.n \rightarrow \infty\right\}$ is an event with probability 1 . We say that $X_{n} \rightarrow X$ in probability, written $X_{n} \stackrel{P}{\rightarrow} X$, if $\mathbb{P}\left(\left|X_{n}-X\right|>\epsilon\right) \rightarrow 0$ as $n \rightarrow \infty$ for all $\epsilon>0$. The following implication holds: $\left(X_{n} \stackrel{\text { a.s. }}{\rightarrow} X\right) \Rightarrow\left(X_{n} \stackrel{\mathrm{P}}{\rightarrow} X\right)$. We shall need the following results.

Theorem 1 (Hoeffding's inequality). Let $X_{1}, \ldots, X_{n}$ be independent random variables. Suppose there exist $a, b \in \mathbb{R}^{n}$ such that $\mathbb{P}\left(X_{i} \in\left[a_{i}, b_{i}\right]\right)=1$. Let $S:=\sum_{j=1}^{n} X_{j}$. Then

$$
\mathbb{P}(S-\mathbb{E} S \geq n t) \leq \exp \left(-\frac{2 n^{2} t^{2}}{\sum_{j=1}^{n}\left(b_{i}-a_{i}\right)^{2}}\right), \quad t>0 .
$$

Theorem 2 (strong law of large numbers). Let $X_{1}, X_{2}, \ldots, X_{n}$ be independent identically distributed random variables. Then

$$
\frac{1}{n} \sum_{j=1}^{n} X_{j} \rightarrow \mu \quad \text { almost surely as } n \rightarrow \infty
$$

for some constant $\mu$, if and only if $\mathbb{E} X_{1}<\infty$. In this case, $\mu=\mathbb{E} X_{1}$.

In the paper, we shall use a weaker version of the strong law of large numbers, which may be stated as follows.

Corollary 1. Let $X_{1}, X_{2}, \ldots, X_{n}$ be independent identically distributed (i.i.d.) random variables, and suppose $\mathbb{E} X_{1}<\infty$. Then

$$
\frac{1}{n} \sum_{j=1}^{n} X_{j} \rightarrow \mathbb{E} X_{1} \quad \text { in probability as } n \rightarrow \infty
$$

\section{Background and problem statement.}

3.1. Background. The asymptotic analysis presented in this paper is based on recent results on global phase-locking obtained by the authors $[10,11]$ (see $[8,9]$ for related work). Before we proceed with stating the problem, we review some relevant results from the aforementioned work. To start with, we define the notion of "critical coupling," as follows.

Definition 1 (critical coupling). Let $n \in \mathbb{N}, n \geq 2$, and let $x \in \mathbb{R}^{n}$ be given. Consider the Kuramoto model of coupled oscillators on the complete graph $K_{n}$ of (1). For this model, we define the critical coupling, $k_{\mathrm{c}}$, as follows:

$$
k_{\mathrm{C}}:=\min \left\{k \geq 0: \exists \phi \in \mathbb{R}^{n} \text { s.t. } \frac{k}{n} \sum_{j=1}^{n} \sin \left(\phi_{j}-\phi_{i}\right)=\langle x\rangle_{n}-x_{i} \text { for all } i\right\} .
$$

In other words, $k_{\mathrm{c}}$ is the smallest nonnegative value of the coupling strength for which the system (1) admits a (global) phase-locked solution. 
We have the following result [10].

Theorem 3. Let $x \in \mathbb{R}^{n}$ be given, and suppose $x_{i} \neq x_{j}$ for some $(i, j)$. Then

$$
2 \frac{1}{n} \sum_{j=1}^{n} \sqrt{1-\left(\frac{x_{j}-\langle x\rangle_{n}}{u}\right)^{2}}=\frac{1}{n} \sum_{j=1}^{n} \frac{1}{\sqrt{1-\left(\frac{x_{j}-\langle x\rangle_{n}}{u}\right)^{2}}}
$$

has a unique solution $u_{n} \in\left(\max _{j}\left|x_{j}-\langle x\rangle_{n}\right|, 2 \max _{j}\left|x_{j}-\langle x\rangle_{n}\right|\right]$, and we have that

$$
k_{\mathrm{C}}=\frac{u_{n}}{\frac{1}{n} \sum_{j=1}^{n} \sqrt{1-\left(\frac{x_{j}-\langle x\rangle_{n}}{u_{n}}\right)^{2}}} .
$$

If $x_{i}=x_{j}$ for all $(i, j)$, we define $u_{n}:=0$ and $k_{\mathrm{C}}:=0$.

Equation (3) expresses the critical coupling $k_{\mathrm{c}}$ in terms of $u_{n}$, the solution to (2). The latter can be evaluated to within user-defined precision with the algorithm described in Appendix A.

3.2. Problem statement. The proof of our main result, which we present in section 4.2, requires several technical steps and is quite involved, but the main idea is easy to follow. Indeed, the idea is to invoke the law of large numbers on the expressions in (2) and (3), which allows one in the limit to pass from sample means to expected values (from summations to integrals). The main technical difficulty with this approach is that, in order to invoke the law of large numbers, one must require the terms under the summation to be independent, while in our case they are not. What saves the argument is the fact that in the limit, we can replace $\langle x\rangle_{n}$ with $\mu$, which gives us the independence we need. Unfortunately, the necessary proofs require a rather notation-heavy technical apparatus. The purpose of the present section is to set up this apparatus and to state the problem formally.

Let $X_{1}, X_{2}, \ldots, X_{n}$ be independent and identically distributed random variables with density function $p: \mathbb{R} \mapsto \mathbb{R}_{\geq 0}$ and expected value $\mu:=\mathbb{E} X_{1}=\int_{\mathbb{R}} x p(x) \mathrm{d} x$. Let $S_{p}:=\{x \in \mathbb{R}$ : $p(x)>0\}$ denote the support of $p$. We assume the following.

Assumption 1. The density function $p$ is (piecewise) continuous and has bounded support. Moreover,

$$
\int_{S_{p}}\left(\frac{u^{2}}{\sqrt{u^{2}-(x-\mu)^{2}}}-2 \sqrt{u^{2}-(x-\mu)^{2}}\right) p(x) \mathrm{d} x<\infty
$$

for all $u \geq \sup _{x \in S_{p}}|x-\mu|$.

Assumption 1 is a technical assumption that will facilitate the forthcoming analysis. The class of distributions satisfying this assumption is sufficiently rich for our present purposes. Note in particular that Assumption 1 is satisfied by all piecewise continuous $p$ such that $\sup _{x \in S_{p}}|x|<\infty$ (bounded support) and $\sup _{x \in S_{p}} p(x)<\infty$ (bounded range). Moreover, since the integrand is a monotone decreasing function of $u$, if follows that if (4) is satisfied for $u=\sup _{x \in S_{p}}|x-\mu|$, then it is satisfied for all $u \geq \sup _{x \in S_{p}}|x-\mu|$. 
Define $D_{n}:=\left\{(x, u, a) \in \mathbb{R}^{n} \times \mathbb{R} \times \mathbb{R}: u>\max _{1 \leq j \leq n}\left|x_{j}-a\right|>0\right\}$, and let $f_{n}: D_{n} \mapsto \mathbb{R}$ and $f:\left[\sup _{x \in S_{p}}|x-\mu|, \infty\right) \mapsto \mathbb{R}$ be given by

$$
\begin{aligned}
f_{n}(x, u, a) & :=\frac{1}{n} \sum_{j=1}^{n} \frac{u^{2}}{\sqrt{u^{2}-\left(x_{j}-a\right)^{2}}}-2 \frac{1}{n} \sum_{j=1}^{n} \sqrt{u^{2}-\left(x_{j}-a\right)^{2}}, \\
f(u) & :=\int_{S_{p}}\left(\frac{u^{2}}{\sqrt{u^{2}-(x-\mu)^{2}}}-2 \sqrt{u^{2}-(x-\mu)^{2}}\right) p(x) \mathrm{d} x .
\end{aligned}
$$

We next define the function $u_{n}: \mathbb{R}^{n} \times \mathbb{R} \mapsto \mathbb{R}$. For all $(x, a) \in \mathbb{R}^{n} \times \mathbb{R}$ such that $\max _{j}\left|x_{j}-a\right|>$ $0, u_{n}(x, a)$ is the unique solution of $f_{n}\left(x, u_{n}(x, a), a\right)=0$. In the case that $\max _{j}\left|x_{j}-a\right|=0$, we define $u_{n}(x, a)=0$.

Let $X$ denote the $n$-tuple of i.i.d. random variables $\left(X_{1}, \ldots, X_{n}\right)$, and let $\langle X\rangle_{n}:=\frac{1}{n} \sum_{j=1}^{n} X_{j}$. The random variables $u_{n}\left(X,\langle X\rangle_{n}\right), u_{n}(X, a)$ (for $a \in \mathbb{R}$ ) are defined in the usual manner, as is the random variable $k_{c}(X)$,

$$
k_{\mathrm{c}}(X):=\frac{u_{n}\left(X,\langle X\rangle_{n}\right)}{\frac{1}{n} \sum_{j=1}^{n} \sqrt{1-\left(\frac{X_{j}-\langle X\rangle_{n}}{u_{n}\left(X,\langle X\rangle_{n}\right)}\right)^{2}}} .
$$

The purpose of this note is to show that, under suitable technical conditions,

$$
u_{n}\left(X,\langle X\rangle_{n}\right) \stackrel{P}{\rightarrow} u^{*},
$$

where

$$
u^{*}:= \begin{cases}f^{-1}(0) & \text { if } f\left(\sup _{x \in S_{p}}|x-\mu|\right)>0 \\ \sup _{x \in S_{p}}|x-\mu| & \text { otherwise, }\end{cases}
$$

It then follows that there exists $k^{*} \geq 0$ such that $k_{\mathrm{c}}(X) \stackrel{P}{\rightarrow} k^{*}$.

\section{A convergence result.}

4.1. Key technical results. We shall now present some technical results that will be helpful in proving our main result. Proofs can be found in Appendix B. We adopt the following notation:

$$
\partial_{u} f_{n}(x, u, a):=\left.\frac{\partial f_{n}(x, \xi, a)}{\partial \xi}\right|_{\xi=u}, \quad \partial_{a} f_{n}(x, u, a):=\left.\frac{\partial f_{n}(x, u, \xi)}{\partial \xi}\right|_{\xi=a},
$$

and

$$
\partial_{a a} f_{n}(x, u, a):=\left.\frac{\partial^{2} f_{n}(x, u, \xi)}{\partial \xi^{2}}\right|_{\xi=a} .
$$

Our first lemma establishes a few useful facts about $f_{n}$ and $u_{n}$.

Lemma 1. The following statements are true for all $n$ :

(i) For all $(x, u, a) \in D_{n}$, we have that $\left|\partial_{a} f_{n}(x, u, a)\right| \leq \sqrt{2}\left|\partial_{u} f_{n}(x, u, a)\right|$.

(ii) For all $(x, u, a) \in D_{n}$, we have that $\partial_{u} f_{n}(x, u, a) \leq-1$ and $\partial_{a a} f_{n}(x, u, a)>0$.

Copyright (C) by SIAM. Unauthorized reproduction of this article is prohibited. 
(iii) Let $c>0$, and suppose $u_{n}\left(x,\langle x\rangle_{n}\right)<(1+c) \max _{j}\left|x_{j}-\langle x\rangle_{n}\right|$. Suppose in addition that $u_{n}(x, \mu)<u_{n}\left(x,\langle x\rangle_{n}\right)$. Then

$$
\left|u_{n}(x, \mu)-u_{n}\left(x,\langle x\rangle_{n}\right)\right|<\left|\mu-\langle x\rangle_{n}\right|+c \max _{j}\left|x_{j}-\langle x\rangle_{n}\right| .
$$

(iv) Let $c>0$, and suppose $u_{n}\left(x,\langle x\rangle_{n}\right) \geq(1+c) \max _{j}\left|x_{j}-\langle x\rangle_{n}\right|$. Then

$$
\left|\partial_{u} f_{n}\left(x, u_{n}\left(x,\langle x\rangle_{n}\right),\langle x\rangle_{n}\right)\right| \leq\left(1-\left(\frac{1}{1+c}\right)^{2}\right)^{-\frac{3}{2}} .
$$

Note that claims (iii) and (iv) of Lemma 1 still hold if we interchange $\langle x\rangle_{n}$ and $\mu$.

Our next lemma shows that $u_{n}$ is continuous in its second argument. This is an important intermediate step because it suggests that, in the limit, we can replace $u_{n}\left(x,\langle x\rangle_{n}\right)$ with $u_{n}(x, \mu)$, enabling us to invoke the law of large numbers (as shown in the forthcoming Propositions 1 and 2).

Lemma 2. For every $\epsilon>0$ there exists $\delta>0$ such that the implication

$$
\left|\mu-\langle x\rangle_{n}\right|<\delta \Rightarrow\left|u_{n}(x, \mu)-u_{n}\left(x,\langle x\rangle_{n}\right)\right|<\epsilon
$$

holds for all $n$.

We have the following proposition.

Proposition 1. Let $X=\left(X_{1}, X_{2}, \ldots, X_{n}\right)$ be an $n$-tuple of i.i.d. random variables with density function $p$, and suppose that $p$ satisfies the conditions of Assumption 1 . Then we have that

$$
u_{n}\left(X,\langle X\rangle_{n}\right)-u_{n}(X, \mu) \stackrel{P}{\rightarrow} 0 .
$$

Proposition 2. Let $X=\left(X_{1}, \ldots, X_{n}\right)$ be an $n$-tuple of i.i.d. random variables with density function $p$, and suppose that $p$ satisfies the conditions of Assumption 1. Then we have that

$$
u_{n}(X, \mu) \stackrel{P}{\rightarrow} u^{*},
$$

where $u^{*}$ is defined at the end of section (3).

4.2. The main result. We are now ready to state our main result, which is essentially a corollary of Propositions 1 and 2 combined.

Theorem 4. Let $X=\left(X_{1}, X_{2}, \ldots, X_{n}\right)$ be an $n$-tuple of i.i.d. random variables with density function $p$, and suppose that $p$ satisfies the conditions of Assumption 1. Then we have that

(a) $u_{n}\left(X,\langle X\rangle_{n}\right) \stackrel{P}{\rightarrow} u^{*}$;

(b) $k_{\mathrm{c}}(X) \stackrel{P}{\rightarrow} u^{*} / \int_{S_{p}} \sqrt{1-\left(\frac{x-\mu}{u^{*}}\right)^{2}} p(x) d x$.

Proof. Combining Propositions 1 and 2, we have that $u_{n}\left(X,\langle X\rangle_{n}\right) \stackrel{P}{\rightarrow} u^{*}$. This proves part (a). To prove part (b), recall that

$$
k_{\mathrm{c}}(X)=\frac{u_{n}\left(X,\langle X\rangle_{n}\right)^{2}}{\frac{1}{n} \sum_{j=1}^{n} \sqrt{u_{n}\left(X,\langle X\rangle_{n}\right)^{2}-\left(X_{j}-\langle X\rangle_{n}\right)^{2}}}
$$

Copyright ( ) by SIAM. Unauthorized reproduction of this article is prohibited. 
when $u_{n}\left(X,\langle X\rangle_{n}\right) \neq 0$, and $k_{c}(X)=0$ otherwise. Under the hypotheses of Assumption 1, we have that $u^{*}>0$ and $\int_{S_{p}} \sqrt{\left(u^{*}\right)^{2}-(x-\mu)^{2}} p(x) \mathrm{d} x>0$. Hence, if we can show that (i) $u_{n}\left(X,\langle X\rangle_{n}\right)^{2} \stackrel{P}{\rightarrow}\left(u^{*}\right)^{2}$, and (ii)

$$
\frac{1}{n} \sum_{j=1}^{n} \sqrt{u_{n}\left(X,\langle X\rangle_{n}\right)^{2}-\left(X_{j}-\langle X\rangle_{n}\right)^{2}} \stackrel{P}{\rightarrow} \int_{S_{p}} \sqrt{\left(u^{*}\right)^{2}-(x-\mu)^{2}} p(x) \mathrm{d} x
$$

then we are done. (If $A \stackrel{P}{\rightarrow} a$ and $B \stackrel{P}{\rightarrow} b$, then $\frac{A}{B} \stackrel{P}{\rightarrow} \frac{a}{b}$, provided $b \neq 0$.) To prove (i), note that for some $c \geq 0$ we have that

$$
\left|u_{n}\left(X,\langle X\rangle_{n}\right)^{2}-\left(u^{*}\right)^{2}\right|=\left|u_{n}\left(X,\langle X\rangle_{n}\right)-u^{*}\right| \cdot\left|u_{n}\left(X,\langle X\rangle_{n}\right)+u^{*}\right| \leq c\left|u_{n}\left(X,\langle X\rangle_{n}\right)-u^{*}\right| .
$$

This implies that

$$
\mathbb{P}\left(\left|u_{n}\left(X,\langle X\rangle_{n}\right)^{2}-\left(u^{*}\right)^{2}\right|>\epsilon\right) \leq \mathbb{P}\left(\left|u_{n}\left(X,\langle X\rangle_{n}\right)-u^{*}\right|>\frac{\epsilon}{c}\right) .
$$

Under the hypotheses of the theorem the right-hand side of inequality (7) tends to zero for every $\epsilon>0$ as $n \rightarrow \infty$ (this was established in part (a)), and hence so does the left-hand side. We conclude that $u_{n}\left(X,\langle X\rangle_{n}\right)^{2} \stackrel{P}{\rightarrow}\left(u^{*}\right)^{2}$. The proof of (ii) requires some more work, but essentially proceeds along the same lines. The idea is to use standard estimates (such as $|\sqrt{a}-\sqrt{b}| \leq \sqrt{|a-b|}$, which holds for all nonnegative $a, b)$ to show that $\langle X\rangle_{n} \stackrel{P}{\rightarrow} \mu$ and $u_{n}\left(X,\langle X\rangle_{n}\right) \stackrel{P}{\rightarrow} u^{*}$ imply that

$$
\frac{1}{n} \sum_{j=1}^{n} \sqrt{u_{n}\left(X,\langle X\rangle_{n}\right)^{2}-\left(X_{j}-\langle X\rangle_{n}\right)^{2}}-\frac{1}{n} \sum_{j=1}^{n} \sqrt{\left(u^{*}\right)^{2}-\left(X_{j}-\mu\right)^{2}} \stackrel{P}{\rightarrow} 0 .
$$

The result then follows from the law of large numbers (Corollary 1). This completes the proof.

Note that in order to evaluate the expression for the critical coupling, we need an estimate for $u^{*}$. In general, this requires solving the integral equation $f(u)=0$. However, in the special case when $f\left(\sup _{x \in S_{p}}|x-\mu|\right) \geq 0, u^{*}$ is simply given as $u^{*}=\sup _{x \in S_{p}}|x-\mu|$. The examples in the next section will deal exclusively with the latter case.

5. Application of the main result. To illustrate the result of Theorem 4 , we present two examples. In the first example, we consider distributions of intrinsic frequencies described by a family of symmetric density functions with finite support that includes the uniform density function. In the second example, we consider a distribution described by an asymmetric density function. The purpose of this example is to show that the application of our result is not limited to symmetric distributions.

5.1. Critical coupling in the limit for a class of symmetric density functions with finite support. Let $\alpha>-\frac{1}{2}, c>0$, and let $p_{\alpha}(x): \mathbb{R} \mapsto \mathbb{R}_{\geq 0}$ be given as

$$
p_{\alpha}(x):= \begin{cases}\frac{1}{c \sqrt{\pi}} \frac{\Gamma\left(\alpha+\frac{3}{2}\right)}{\Gamma(\alpha+1)}\left(1-\left(\frac{x-\mu}{c}\right)^{2}\right)^{\alpha}, & |x-\mu|<c, \\ 0 & |x-\mu| \geq c .\end{cases}
$$

Copyright ( $\odot$ by SIAM. Unauthorized reproduction of this article is prohibited. 


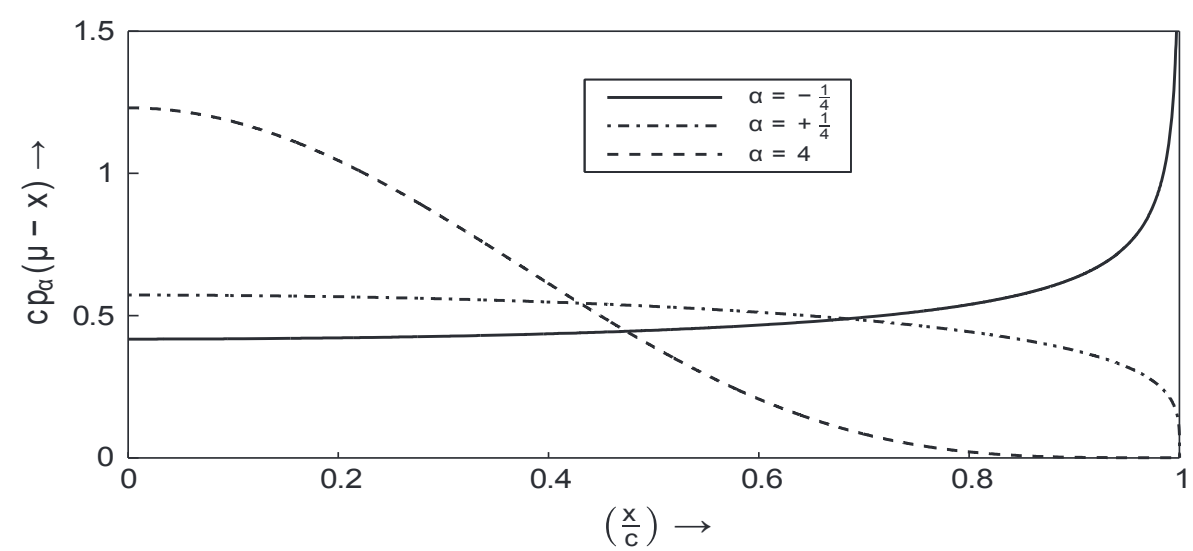

Figure 1. The graph of $p_{\alpha}(\mu-x)$ on the interval $[0, c]$ for $\alpha \in\left\{-\frac{1}{4}, \frac{1}{4}, 4\right\}$.

Note that $\mathbb{E}_{\alpha}(x):=\int_{\mathbb{R}} x p_{\alpha}(x) \mathrm{d} x=\mu$, and that $p_{\alpha}(\mu-x)=p_{\alpha}(\mu+x)$ for all $x$. In other words, $p_{\alpha}$ is symmetric about its mean, $\mu$. Figure 1 shows the graph of $p_{\alpha}(\mu-x)$ on the interval $[0, c]$ for selected values of $\alpha$. Note that $p_{0}$ is the uniform density function on $(-c, c)$. For all $\alpha$ we have that $S_{p_{\alpha}}=(-c, c)$ and $\sup _{x \in S_{p_{\alpha}}}|x-\mu|=c$. For the family of distributions considered here we can evaluate $f\left(\sup _{x \in S_{p_{\alpha}}}|x-\mu|\right)$ analytically. Indeed, we have that

$$
f\left(\sup _{x \in S_{p_{\alpha}}}|x-\mu|\right):= \begin{cases}-\left(\frac{2}{\alpha(\alpha+1)(1+2 \alpha)} \cdot \frac{\Gamma^{2}\left(\alpha+\frac{3}{2}\right)}{\Gamma^{2}(\alpha)}\right) c, & \alpha>-\frac{1}{2}, \alpha \neq 0 \\ 0, & \alpha=0,\end{cases}
$$

where $\Gamma(\cdot)$ is the Gamma function. It follows that $f\left(\sup _{x \in S_{p_{\alpha}}}|x-\mu|\right)>0$ if and only if $\alpha<0$. By Theorem 4, this implies that for all $\alpha \geq 0$ we have that

$$
k_{\mathrm{c}}(X) \stackrel{P}{\rightarrow} \frac{c}{\int_{S_{p}} \sqrt{1-\left(\frac{x-\mu}{c}\right)^{2}} p(x) \mathrm{d} x}=\frac{\Gamma(\alpha+1) \Gamma(\alpha+2)}{\Gamma^{2}\left(\alpha+\frac{3}{2}\right)} c .
$$

In particular, for $\alpha=0$ we have that $K_{\mathrm{c}} \stackrel{P}{\rightarrow} \frac{4}{\pi} c=\frac{2}{\pi p_{0}(\mu)}$, which coincides with the Kuramoto coupling value for the onset of partially phase-locked behavior. Figure 2 shows how, for the case $c=\frac{1}{2}, \alpha=0$, both the critical coupling,

$$
k_{\mathrm{c}}(x):=\frac{u_{n}\left(x,\langle x\rangle_{n}\right)}{\frac{1}{n} \sum_{j=1}^{n} \sqrt{1-\left(\frac{x_{j}-\langle x\rangle_{n}}{u_{n}\left(x,\langle x\rangle_{n}\right)}\right)^{2}}}
$$

and the estimate obtained by replacing $\langle x\rangle_{n}$ with $\mu$,

$$
\hat{k}_{\mathrm{c}}(x):=\frac{u_{n}(x, \mu)}{\frac{1}{n} \sum_{j=1}^{n} \sqrt{1-\left(\frac{x_{j}-\mu}{u_{n}(x, \mu)}\right)^{2}}},
$$

converge to the expected value $\mathbb{E}_{X} k_{\mathrm{c}}(X)=\frac{2}{\pi}$ as the number of oscillators increases. Figure 3 


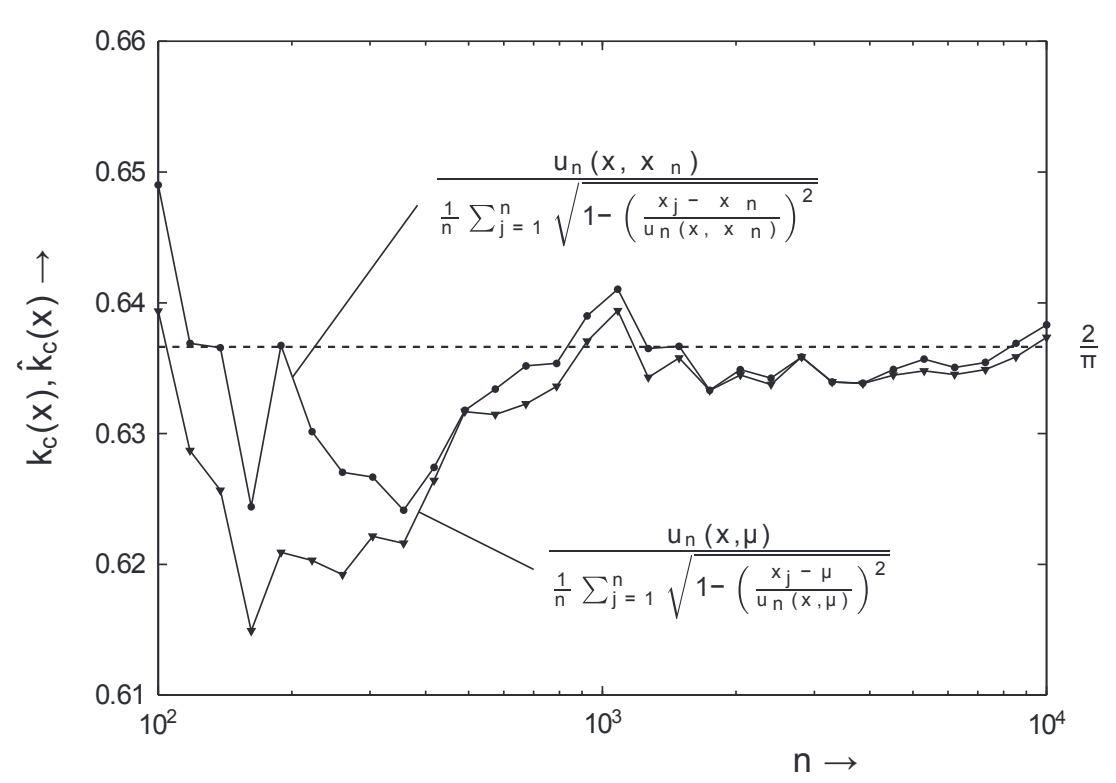

Figure 2. The graph of $k_{\mathrm{c}}$ and $\hat{k}_{\mathrm{c}}$, (9) and (10), for a given realization of intrinsic frequencies $x=$ $\left(x_{1}, \ldots, x_{n}\right)$ and selected values of $n$ in the interval $\left[10^{2}, 10^{4}\right]$.

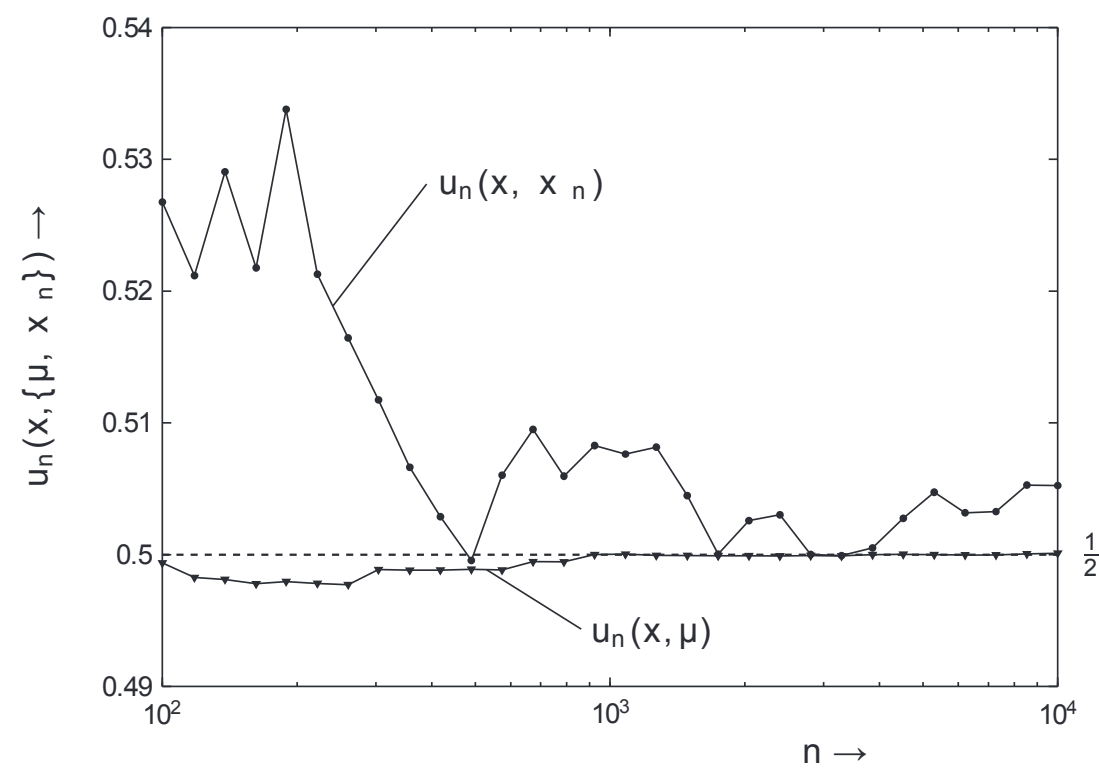

Figure 3. The graph of $u_{n}\left(x, x_{n}\right)$ and $u_{n}(x, \mu)$ for a particular realization of intrinsic frequencies $x=$ $\left(x_{1}, \ldots, x_{n}\right)$ and selected values of $n$ in the interval $\left[10^{2}, 10^{4}\right]$.

shows, again for the case $c=\frac{1}{2}, \alpha=0$, the behavior of $u_{n}\left(x,\langle x\rangle_{n}\right)$ and $u_{n}(x, \mu)$ as a function of $n$. Note that $u_{n}(x, \mu)$ appears to converge much faster than $u_{n}\left(x,\langle x\rangle_{n}\right)$. For $\alpha>0$, we have that

$$
k_{\mathrm{c}}(X) \stackrel{P}{\rightarrow}\left(\frac{\frac{1}{2} \sqrt{\pi} \cdot \Gamma(\alpha+2)}{\Gamma\left(\alpha+\frac{3}{2}\right)}\right) \frac{2}{\pi p_{\alpha}(\mu)}
$$

Copyright (C) by SIAM. Unauthorized reproduction of this article is prohibited. 
which suggests that the relative difference between the Kuramoto coupling and the critical coupling in the sense of Definition 1 diverges as $\alpha$ tends to infinity, with the former (associated with the onset of global phase-locking) tending to $c$ and the latter (associated with the onset of partial phase-locking) tending to 0 .

\subsection{Critical coupling in the limit for a nonsymmetric distribution with finite support.} Next we consider a simple nonsymmetric density function $p: \mathbb{R} \mapsto \mathbb{R}_{\geq 0}$,

$$
p(x):= \begin{cases}2-2 x & \text { for } 0<x \leq 1 \\ 0 & \text { elsewhere }\end{cases}
$$

For this distribution we have that $\mu=\frac{1}{3}, S_{p}=(0,1)$, and $\sup _{x \in S_{p}}|x-\mu|=\frac{2}{3}$. Inspection shows that

$$
f\left(\sup _{x \in S_{p}}|x-\mu|\right)=f\left(\frac{2}{3}\right)=-\frac{8}{27} \sqrt{3}
$$

Hence, by Theorem 2, we have that

$$
k_{\mathrm{c}}(X) \stackrel{P}{\rightarrow} \frac{\sup _{x \in S_{p}}|x-\mu|}{\int_{S_{p}} \sqrt{1-\left(\frac{x-\mu}{\sup _{x \in S_{p}}|x-\mu|}\right)^{2}} p(x) \mathrm{d} x}=\frac{\frac{2}{3}}{\frac{8}{27} \pi}=\frac{9}{4 \pi} .
$$

This is consistent with simulation results (data not shown).

6. Conclusion. In this paper we proved a convergence result for the Kuramoto model. In particular, we showed that, under appropriate technical conditions on the distribution of intrinsic frequencies, the critical coupling strength associated with the emergence of global phase-locked solutions converges in probability as the number of oscillators tends to infinity. In general, this critical value differs from the Kuramoto coupling, which relates to the existence of partially phase-locked stationary solutions, but for some distributions they coincide (notably for the uniform distribution). We hope that the results described in this paper contribute to a better understanding of the asymptotic properties of the classical Kuramoto model.

Appendix A. Algorithm for computing the critical coupling. The algorithm below computes the solution $u_{n}$ to (2) for a given vector $x=\left(x_{1}, x_{2}, \ldots, x_{n}\right)$ of intrinsic frequencies, with precision AbsTol.

1. $a:=\max _{j}\left|x_{j}-\langle x\rangle_{n}\right| ;$

2. $u:=2 \cdot a$;

3. AbsTol : $=10^{-6}$;

4. $\operatorname{Err}:=1$

5. $\Delta_{\mathrm{u}}:=\frac{1}{2}(\mathrm{u}-\mathrm{a})$;

6. while $|\operatorname{Err}|>$ AbsTol

$$
\begin{aligned}
& \text { 6.1. } \operatorname{Err}:=\sum_{j} \sqrt{1-\left(\frac{x_{j}-\langle x\rangle_{n}}{u}\right)^{2}}-\frac{1}{2} \sum_{j} \frac{1}{1-\left(\frac{x_{j}-\langle x\rangle_{n}}{u}\right)^{2}} ; \\
& \text { 6.2. if } \operatorname{Err} \geq 0
\end{aligned}
$$


$\begin{aligned} \mathrm{u} & :=\mathrm{u}-\Delta_{\mathrm{u}} \\ \Delta_{\mathrm{u}} & :=\end{aligned}$

6.3. else

6.4. end

$\mathrm{u}:=\mathrm{u}+\Delta_{\mathrm{u}}$

7. end

\section{Appendix B. Proofs.}

\section{B.1. Proof of Lemma 1.}

Proof. To prove Lemma 1(i), let $(x, u, a) \in D_{n}$. Then by definition we have that $u>$ $\max _{j}\left|x_{j}-a\right|$, and the result follows by inspection:

$$
\begin{aligned}
\left|\partial_{a} f_{n}(x, u, a)\right| & =\left|\frac{1}{n} \sum_{j=1}^{n}\left(\frac{x_{j}-a}{u}\right)\left(3-2\left(\frac{x_{j}-a}{u}\right)^{2}\right)\left(1-\left(\frac{x_{j}-a}{u}\right)^{2}\right)^{-\frac{3}{2}}\right| \\
& \leq \max _{j}\left|\left(\frac{x_{j}-a}{u}\right)\left(3-2\left(\frac{x_{j}-a}{u}\right)^{2}\right)\right| \frac{1}{n} \sum_{j=1}^{n}\left(1-\left(\frac{x_{j}-a}{u}\right)^{2}\right)^{-\frac{3}{2}} \\
& \leq \sqrt{2} \underbrace{\frac{1}{n} \sum_{j=1}^{n}\left(1-\left(\frac{x_{j}-a}{u}\right)^{2}\right)^{-\frac{3}{2}}}_{=:-\partial_{u} f_{n}(x, u, a)} .
\end{aligned}
$$

Part (ii) again follows by inspection. Indeed, we have that

$$
\begin{gathered}
\partial_{u} f_{n}(x, u, a)=-\frac{1}{n} \sum_{j=1}^{n}\left(1-\left(\frac{x_{j}-a}{u}\right)^{2}\right)^{-\frac{3}{2}} \leq-1, \\
\partial_{a a} f_{n}(x, u, a)=\frac{1}{n} \sum_{j=1}^{n}\left(\frac{3}{u}\right)\left(1-\left(\frac{x_{j}-a}{u}\right)^{2}\right)^{-\frac{5}{2}}>0 .
\end{gathered}
$$

To prove part (iii), note that, under the given hypotheses,

$$
\max _{j}\left|x_{j}-\mu\right|<u_{n}(x, \mu)<u_{n}\left(x,\langle x\rangle_{n}\right)<(1+c) \max _{j}\left|x_{j}-\langle x\rangle_{n}\right| .
$$

This implies that

$$
\begin{aligned}
\left|u_{n}(x, \mu)-u_{n}\left(x,\langle x\rangle_{n}\right)\right| & <(1+c) \max _{j}\left|x_{j}-\langle x\rangle_{n}\right|-\max _{j}\left|x_{j}-\mu\right| \\
& =\max _{j}\left|x_{j}-\langle x\rangle_{n}\right|-\max _{j}\left|x_{j}-\mu\right|+c \max _{j}\left|x_{j}-\langle x\rangle_{n}\right| \\
& \leq\left|\mu-\langle x\rangle_{n}\right|+c \max _{j}\left|x_{j}-\langle x\rangle_{n}\right|,
\end{aligned}
$$

Copyright (C) by SIAM. Unauthorized reproduction of this article is prohibited. 
as required. Finally, let $c>0$ and suppose $u_{n}\left(x,\langle x\rangle_{n}\right) \geq(1+c) \max _{j}\left|x_{j}-\langle x\rangle_{n}\right|$. Part (iv) follows by inspection:

$$
\begin{aligned}
\left|\partial_{u} f_{n}\left(x, u_{n}\left(x,\langle x\rangle_{n}\right),\langle x\rangle_{n}\right)\right| & :=\left|\frac{1}{n} \sum_{j=1}^{n}\left(1-\left(\frac{x_{j}-\langle x\rangle_{n}}{u_{n}\left(x,\langle x\rangle_{n}\right)}\right)^{2}\right)^{-\frac{3}{2}}\right| \\
& \leq \max _{j}\left(1-\left(\frac{x_{j}-\langle x\rangle_{n}}{u_{n}\left(x,\langle x\rangle_{n}\right)}\right)^{2}\right)^{-\frac{3}{2}}=\left(1-(1+c)^{-2}\right)^{-\frac{3}{2}}
\end{aligned}
$$

This concludes the proof.

\section{B.2. Proof of Lemma 2.}

Proof. Define $\lambda:=\sup _{x, y \in S_{p}}|x-y|$, and note that $\lambda<\infty$ by Assumption 1. We distinguish two cases: (i) $x_{i}=x_{j}$ for all $(i, j)$; (ii) $x_{i} \neq x_{j}$ for some $(i, j)$. To prove case (i), let $\epsilon>0$ be given, and define $\delta:=\epsilon$. Under the given hypotheses, it follows from the definition of $u_{n}$ that $u_{n}(x, \mu)=\left|\mu-\langle x\rangle_{n}\right|$ and $u_{n}\left(x,\langle x\rangle_{n}\right)=0$. Now suppose $\left|\mu-\langle x\rangle_{n}\right|<\delta$. Then by construction we have that

$$
\left|u_{n}(x, \mu)-u_{n}\left(x,\langle x\rangle_{n}\right)\right|=\left|\mu-\langle x\rangle_{n}\right|<\delta=\epsilon,
$$

as required. To prove case (ii), let $x_{i} \neq x_{j}$ for some $(i, j)$ and suppose $u_{n}\left(x,\langle x\rangle_{n}\right)>u_{n}(x, \mu)$. (In the case $u_{n}\left(x,\langle x\rangle_{n}\right)=u_{n}(x, \mu)$ there is nothing to prove, while in case $u_{n}\left(x,\langle x\rangle_{n}\right)<$ $u_{n}(x, \mu)$ the result follows by analogy with the present case.) Let $\epsilon>0$ be given, and define $\delta:=\frac{1}{2} \epsilon \min \left\{1, \sqrt{2}\left(1-\left(1+\frac{\epsilon}{2 \lambda}\right)^{-2}\right)^{\frac{3}{2}}\right\}$. We distinguish two cases:

$$
\begin{cases}(\text { ii-a }) & u_{n}\left(x,\langle x\rangle_{n}\right)<\left(1+\frac{\epsilon}{2 \lambda}\right) \max _{j}\left|x_{j}-\langle x\rangle_{n}\right| . \\ \text { (ii-b) } & u_{n}\left(x,\langle x\rangle_{n}\right) \geq\left(1+\frac{\epsilon}{2 \lambda}\right) \max _{j}\left|x_{j}-\langle x\rangle_{n}\right| .\end{cases}
$$

First we consider case (ii-a). Suppose $\left|\mu-\langle x\rangle_{n}\right|<\delta$. Under the given hypotheses, it follows from Lemma 1(iii) that

$$
\left|u_{n}(x, \mu)-u_{n}\left(x,\langle x\rangle_{n}\right)\right|<\left|\mu-\langle x\rangle_{n}\right|+\frac{\epsilon}{2 \lambda} \max _{j}\left|x_{j}-\langle x\rangle_{n}\right|<\frac{1}{2} \epsilon+\frac{1}{2} \epsilon=\epsilon,
$$

as required. Next we consider case (ii-b). First, note that under the given hypotheses $f_{n}(x, \cdot, \mu)$ is strictly concave and differentiable on $\left(\max _{1 \leq j \leq n}\left|x_{j}-\mu\right|, \infty\right)$. This implies that

$$
0<u_{n}\left(x,\langle x\rangle_{n}\right)-u_{n}(x, \mu) \leq\left|\frac{f_{n}\left(x, u_{n}\left(x,\langle x\rangle_{n}\right), \mu\right)}{\partial_{u} f_{n}\left(x, u_{n}\left(x,\langle x\rangle_{n}\right), \mu\right)}\right|<\infty
$$

(see Figure 4 for an illustration). Second, note that, when restricted to the interval $I:=$ $\left[\min \left\{\mu,\langle x\rangle_{n}\right\}, \max \left\{\mu,\langle x\rangle_{n}\right\}\right]$, the function $f_{n}\left(x, u_{n}\left(x,\langle x\rangle_{n}\right), \cdot\right)$ is Lipschitz. In particular, we 


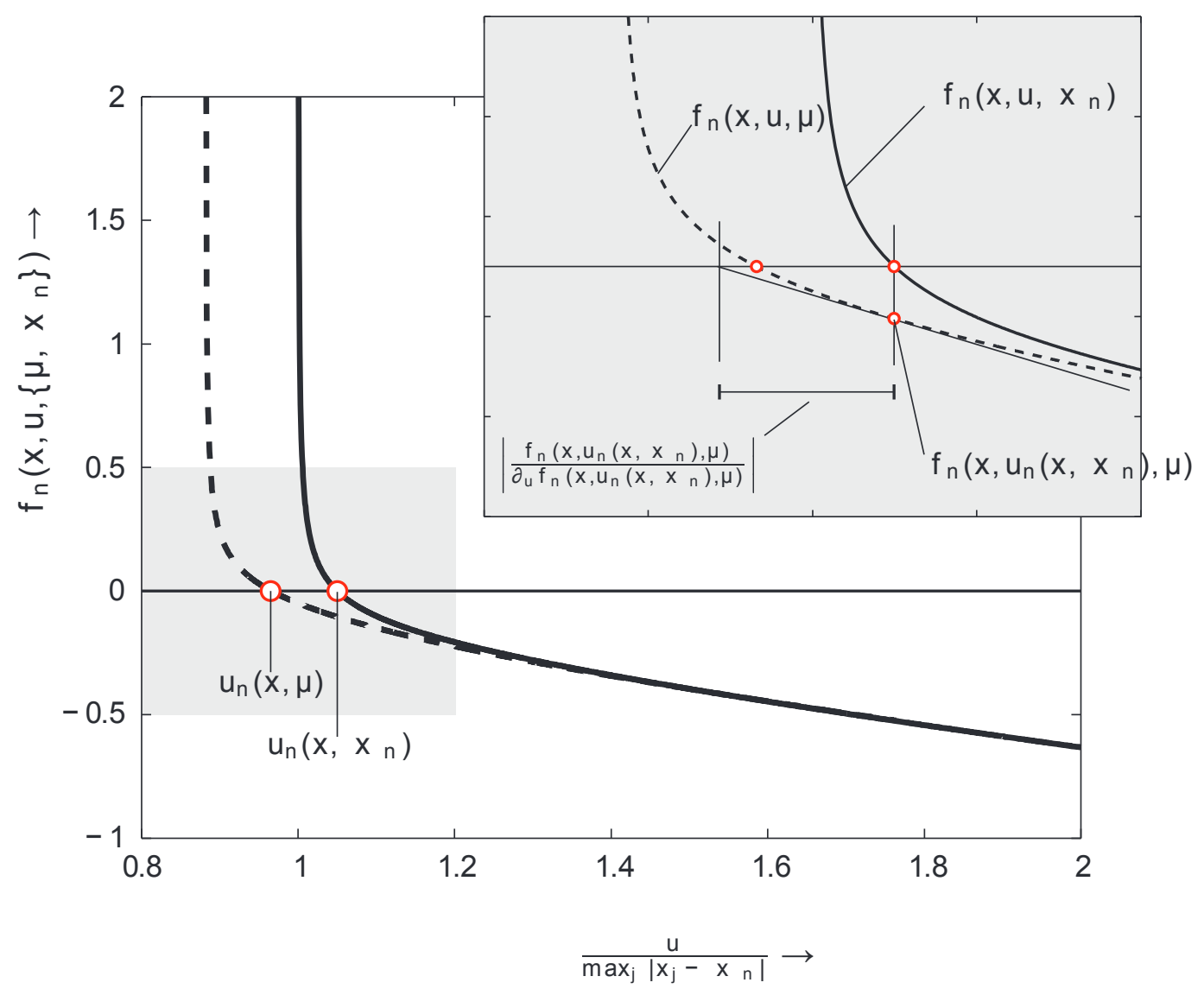

Figure 4. Illustration with the proof of Lemma 2.

have that

$$
\left|f_{n}\left(x, u_{n}\left(x,\langle x\rangle_{n}\right), \mu\right)-f_{n}\left(x, u_{n}\left(x,\langle x\rangle_{n}\right),\langle x\rangle_{n}\right)\right|<\max _{a \in I}\left|\partial_{a} f_{n}\left(x, u_{n}\left(x,\langle x\rangle_{n}\right), a\right)\right|\left|\mu-\langle x\rangle_{n}\right| .
$$

Since $\partial_{a a} f_{n}\left(x, u_{n}\left(x,\langle x\rangle_{n}\right), a\right)>0$ by Lemma 1(ii), it follows that $\max _{a \in I} \mid \partial_{a} f_{n}\left(x, u_{n}\left(x,\langle x\rangle_{n}\right)\right.$, $a)\left|=\max _{a \in\left\{\mu,\langle x\rangle_{n}\right\}}\right| \partial_{a} f_{n}\left(x, u_{n}\left(x,\langle x\rangle_{n}\right), a\right) \mid$. Now recall that $f_{n}\left(x, u_{n}\left(x,\langle x\rangle_{n}\right),\langle x\rangle_{n}\right)=0$ by definition of $u_{n}\left(x,\langle x\rangle_{n}\right)$. It follows that

$$
\left|u_{n}\left(x,\langle x\rangle_{n}\right)-u_{n}(x, \mu)\right| \leq \max _{a \in\left\{\mu,\langle x\rangle_{n}\right\}}\left|\frac{\partial_{a} f_{n}\left(x, u_{n}\left(x,\langle x\rangle_{n}\right), a\right)}{\partial_{u} f_{n}\left(x, u_{n}\left(x,\langle x\rangle_{n}\right), \mu\right)}\right|\left|\mu-\langle x\rangle_{n}\right| .
$$

Direct application of Lemma 1 parts (i), (ii), and (iv) yields

$$
\begin{aligned}
\left|\partial_{a} f_{n}\left(x, u_{n}\left(x,\langle x\rangle_{n}\right), \mu\right)\right| & \leq \sqrt{2}\left|\partial_{u} f_{n}\left(x, u_{n}\left(x,\langle x\rangle_{n}\right), \mu\right)\right|, \\
\left|\partial_{u} f_{n}\left(x, u_{n}\left(x,\langle x\rangle_{n}\right), \mu\right)\right| & \geq 1 \\
\left|\partial_{u} f_{n}\left(x, u_{n}\left(x,\langle x\rangle_{n}\right),\langle x\rangle_{n}\right)\right| & \leq\left(1-\left(1+\frac{\epsilon}{2 \lambda}\right)^{-2}\right)^{-\frac{3}{2}}
\end{aligned}
$$

Copyright (C) by SIAM. Unauthorized reproduction of this article is prohibited. 
Combined with (11) this gives us

$$
\begin{aligned}
\left|u_{n}\left(x,\langle x\rangle_{n}\right)-u_{n}(x, \mu)\right| & \leq \sqrt{2} \max \left\{1,\left(1-\left(1+\frac{\epsilon}{2 \lambda}\right)^{-2}\right)^{-\frac{3}{2}}\right\}\left|\mu-\langle X\rangle_{n}\right| \\
& =\sqrt{2}\left(1-\left(1+\frac{\epsilon}{2 \lambda}\right)^{-2}\right)^{-\frac{3}{2}}\left|\mu-\langle x\rangle_{n}\right| .
\end{aligned}
$$

Now in conclusion suppose $\left|\mu-\langle x\rangle_{n}\right|<\delta$. Then by construction we have that $\mid u_{n}(x, \mu)-$ $u_{n}\left(x,\langle x\rangle_{n}\right) \mid<\epsilon$. This proves the case $u_{n}(x, \mu)<u_{n}\left(x,\langle x\rangle_{n}\right)$. To prove the case $u_{n}(x, \mu)>$ $u_{n}\left(x,\langle x\rangle_{n}\right)$, interchange $\mu$ and $\langle x\rangle_{n}$ as suggested by the discussion following Lemma 1. Inspection shows that all the inequalities still hold. This concludes the proof.

\section{B.3. Proof of Proposition 1.}

Proof. What we need to show is that, for all $\epsilon>0$,

$$
\mathbb{P}\left(\left|u_{n}\left(X,\langle X\rangle_{n}\right)-u_{n}(X, \mu)\right|>\epsilon\right) \rightarrow 0 \quad \text { as } n \rightarrow \infty .
$$

Let $\epsilon>0$, and define $\delta(\epsilon):=\frac{1}{2} \epsilon \min \left\{1, \sqrt{2}\left(1-\left(1+\frac{\epsilon}{2 \lambda}\right)^{-2}\right)^{\frac{3}{2}}\right\}$, where, as before, $\lambda:=\sup _{x, y \in S_{p}}$ $|x-y|$. Then by Lemma 2 we have that

$$
\mathbb{P}\left(\left|u_{n}\left(X,\langle X\rangle_{n}\right)-u_{n}(X, \mu)\right|>\epsilon\right) \leq \mathbb{P}\left(\left|\mu-\langle X\rangle_{n}\right| \geq \delta(\epsilon)\right) .
$$

Moreover, by Hoeffding's inequality (Theorem 1), we have that

$$
\mathbb{P}\left(\left|\mu-\langle X\rangle_{n}\right| \geq \delta(\epsilon)\right) \leq \exp \left(\frac{-2 n \delta(\epsilon)^{2}}{\lambda}\right) .
$$

This implies that

$$
\mathbb{P}\left(\left|u_{n}\left(X,\langle X\rangle_{n}\right)-u_{n}(X, \mu)\right|>\epsilon\right) \leq \exp \left(\frac{-2 n \delta(\epsilon)^{2}}{\lambda}\right) .
$$

We observe that the right-hand side of (12) tends to 0 as $n \rightarrow \infty$ and does so for every $\epsilon>0$. We conclude that $u_{n}\left(X,\langle X\rangle_{n}\right)-u_{n}(X, \mu) \stackrel{P}{\rightarrow} 0$.

B.4. Proof of Proposition 2.

Proof. Let $f$ be given by (5). Observe that $f$ is strictly decreasing:

$$
\left.\frac{\partial f(\xi)}{\partial \xi}\right|_{\xi=u}=-\int_{S_{p}}\left(1-\left(\frac{x-\mu}{u}\right)^{2}\right)^{-\frac{3}{2}} p(x) \mathrm{d} x<0 .
$$

Suppose $f\left(\sup _{x \in S_{p}}|x-\mu|\right)>0$. Since $f(u)<0$ for large $u$, by continuity there exists $u^{\prime}>\sup _{x \in S_{p}}|x-\mu|$ such that $f\left(u^{\prime}\right)=0$. Let $\epsilon>0$ be small (in particular, let $\epsilon<u^{\prime}-$ $\left.\max _{x \in S_{p}}|x-\mu|\right)$. Then by Corollary 1 , noting that

$$
Z_{i}:=\frac{u^{2}}{\sqrt{u^{2}-X_{i}^{2}}}-2 \sqrt{u^{2}-X_{i}^{2}}, \quad i=1, \ldots, n,
$$


are i.i.d. random variables, we have that $f_{n}\left(X, u^{\prime}-\epsilon, \mu\right) \stackrel{P}{\rightarrow} f\left(u^{\prime}-\epsilon\right)>0$ and $f_{n}\left(X, u^{\prime}+\epsilon, \mu\right) \stackrel{P}{\rightarrow}$ $f\left(u^{\prime}+\epsilon\right)<0$. By continuity of $f$ and $f_{n}$ this implies that $\mathbb{P}\left(\left|u^{\prime}-u_{n}(X, \mu)\right|>\epsilon+\delta\right) \rightarrow 0$ for all $\delta>0$ as $n \rightarrow \infty$. Since this is true for every $\epsilon$ it follows that $u_{n}(X, \mu) \stackrel{P}{\rightarrow} u^{\prime}$. Note that $u^{\prime}=u^{*}$ by definition. Now suppose instead $f\left(\sup _{x \in S_{p}}|x-\mu|\right) \leq 0$, and define $u^{\prime}:=\sup _{x \in S_{p}}|x-\mu|$. Let $\epsilon>0$. Again by Corollary 1 we have that $f_{n}\left(u^{\prime}+\epsilon, \mu\right) \stackrel{P}{\rightarrow} f\left(u^{\prime}+\epsilon\right)<0$. By the same argument as above, this implies that $\mathbb{P}\left(u_{n}(X, \mu)-u^{\prime}>\delta+\epsilon\right) \rightarrow 0$ for all $\delta>0$ and all $\epsilon>0$ as $n \rightarrow \infty$. What remains to be shown is that $\mathbb{P}\left(u_{n}(X, \mu)-u^{\prime}<-\delta\right) \rightarrow 0$ for all $\delta>0$ as $n \rightarrow \infty$. Recall that $u_{n}(X, \mu)>\max _{j}\left|X_{j}-\mu\right|$. It is easy to see that, under the conditions of Assumption $1, \mathbb{P}\left(\max _{j}\left|X_{j}-\mu\right|-u^{\prime}<-\delta\right) \rightarrow 0$ for all $\delta>0$ as $n \rightarrow \infty$. This implies that $\mathbb{P}\left(u_{n}(X, \mu)-u^{\prime}<-\delta\right) \rightarrow 0$ for all $\delta>0$ as $n \rightarrow \infty$, as required. Note that $u^{\prime}=u^{*}$ by definition. This concludes the proof.

\section{REFERENCES}

[1] Y. Kur a mot o, Self-entrainment of a population of coupled nonlinear oscillators, in Proceedings of the International Symposium on Mathematical Problems in Theoretical Physics, H. Araki, ed., Lecture Notes in Phys. 39, Springer, New York, 1975, pp. 420-442.

[2] Y. Kur a mot o, Chemical Oscillations, Waves and Turbulence, Springer, New York, 1984.

[3] S. St r ogat z, From Kuramoto to Crawford: Exploring the onset of synchronization in populations of coupled oscillators, Phys. D, 143 (2000), pp. 1-20.

[4] J. Acebrón, L. Bonilla, C. Pérez Vicente, F. Rit ort, and R. Spigler, The Kuramoto model: A simple paradigm for synchronization phenomena, Rev. Modern Phys., 77 (2005), pp. 137-185.

[5] Y. Maist renko, V. Popovych, O. Burylko, and P. Tass, Mechanism of desynchronization in the finite-dimensional Kuramoto model, Phys. Rev. Lett., 93 (2004), 084102.

[6] Y. Maist renko, O. Popovych, and P. Tass, Desynchronization and Chaos in the Kuramoto Model, Lecture Notes in Phys. 671, Springer, Berlin, 2005.

[7] D. Quinn, R. Rand, and S. St rogat z, Singular unlocking transition in the Winfre model of coupled oscillators, Phys. Rev. E, 75 (2007), pp. 36218-36227.

[8] R. Mirollo and S. Strogat z, The spectrum of the locked state for the Kuramoto model of coupled oscillators, Phys. D, 205 (2005), pp. 249-266.

[9] R. Mirollo and S. Strogat z, The spectrum of the partially locked state for the Kuramoto model, J. Nonlinear Sci., 7 (2007), pp. 309-347.

[10] M. Verwoerd and O. Mason, Global phase-locking in finite populations of phase-coupled oscillators, SIAM J. Appl. Dynam. Syst., 7 (2008), pp. 134-160.

[11] M. Verwoerd and O. Mason, On computing the critical coupling coeffi cient for the Kuramoto model on a complete bipartite graph, SIAM J. Appl. Dynam. Syst., 8 (2009), pp. 417-453.

[12] G. Grimmet t and D. St ir zaker, Probability and Random Processes, 3rd ed., Oxford University Press, London, 2005.

[13] S. St rogat z and R. Mirollo, Stability of incoherence in a population of coupled oscillators, J. Statist. Phys., 63 (1991), pp. 613-635.

Copyright (C) by SIAM. Unauthorized reproduction of this article is prohibited. 
